\title{
Effects of 4-1BB signaling on the biological function of murine dendritic cells
}

\author{
YOULIN KUANG, XIAODONG WENG, XIUHENG LIU, HENGCHEN ZHU, ZHIYUAN CHEN and HUI CHEN
}

Department of Urology, Renmin Hospital of Wuhan University, Wuhan 430060, P.R. China

Received August 3, 2011; Accepted November 14, 2011

DOI: $10.3892 / 01.2011 .506$

\begin{abstract}
BB signaling has profound effects on the T cellinduced cell immune response, but its biological function in dendritic cells (DCs) has remained largely uncharacterized. In this study, we investigated the function of 4-1BB in murine DCs with an agonistic mAb to 4-1BB. Interleukin (IL)-6 and IL-12 production was assessed by an enzyme-linked immunosorbent assay (ELISA). Co-stimulatory molecules (CD80 and CD86) in DCs were analyzed by flow cytometry. The results showed that 4-1BB was strongly expressed in DCs during the maturation process. Triggering 4-1BB increased the secretion of IL-6 and IL-12 and the upregulation of co-stimulatory molecules (CD80 and CD86) from DCs, indicating that agonistic mAb to 4-1BB directly improves the activation of DCs. Moreover, triggering 4-1BB induced a higher survival rate of DCs compared to that of hamster IgG isotype control, due to the upregulated expression of Bcl-2 and Bcl-xL. To further assess the role of 4-1BB on DCs stimulating T-cell proliferation, allogeneic mixed lymphocyte reactions were analyzed. The agonistic anti-4-1BB mAb induced a higher T-cell proliferation. These results suggest that $4-1 \mathrm{BB}$ affects the duration, DC-T interaction and immunogenicity of DCs.
\end{abstract}

\section{Introduction}

Dendritic cells (DCs) are potent antigen-presenting cells that play a central role in immunity (1). Following antigen uptake in the peripheral tissue, immature DCs migrate to the secondary lymphoid organs where they interact with $\mathrm{T}$ cells, undergoing maturation characterized by an increased ability to process and present antigenic peptides, and a simultaneous decrease in their ability to phagocytose Ags (2). Following maturation, DCs upregulate the expression of both MHC and co-stimulatory molecules, and downmodulate anti-apoptotic

Correspondence to: Professor Xiuheng Liu, Department of Urology, Renmin Hospital of Wuhan University, 238 Jiefang Road, Wuhan 430060, P.R. China

E-mail:1xh670@163.com

Key words: dendritic cells, co-stimulatory molecules, 4-1BB molecules to regulate an Ag-specific immune response simultaneously (3).

4-1BB is a TNFR superfamily member expressed by activated $\mathrm{T}$ lymphocytes (4). Its activation in $\mathrm{T}$ cells enhances T-cell proliferation, long-term survival, the anti-apoptosis of activation-induced $\mathrm{CD}^{+} \mathrm{T}$ cells $(5)$ and the release of T-helper type 1 (Th1) cytokines such as IFN- $\gamma$ and IL-2 $(5,8)$. The systemic treatment of mAbs against $4-1 \mathrm{BB}$ or gene transfer of the 4-1BB ligand into tumor cells induces marked cell-mediated immune responses against tumors $(9,10)$. The administration of anti-4-1BB mAb in tumor-bearing mice leads to the regression of established tumors in a number of mouse models (11). 4-1BB-mediated signaling plays a significant role in T-cell activation and $\mathrm{T}$ cell-mediated immune response, which is well characterized; however, its role on DCs is less well understood.

To further characterize the function of $4-1 \mathrm{BB}$ in DCs, we used an agonistic $\mathrm{mAb}$ against $4-1 \mathrm{BB}$ to trigger $4-1 \mathrm{BB}$ signaling and detected its immunoactivity in dendritic cells.

\section{Materials and methods}

Animals, cell lines and antibodies. Female C57BL/6 (H-2 K $\left.{ }^{b}\right)$ mice, 6-8 weeks old, were obtained from Shanghai SLAC Laboratory Animal Co., Ltd. (Shanghai, China). Animals were maintained at the Central Animal Facility of Wuhan University according to standard guidelines, and experiments were conducted according to the guidelines of the China Council for Animal Care. Cells were cultured in RPMI-1640 medium with $10 \%$ fetal calf serum (FCS), 2 mM L-glutamine, $100 \mathrm{U} / \mathrm{ml}$ penicillin and $100 \mu \mathrm{g} / \mathrm{ml}$ streptomycin at $37^{\circ} \mathrm{C}$ in a humidified atmosphere containing $5 \% \mathrm{CO}_{2}$.

Anti-4-1BB mAb (clone 158,321) was purchased from R\&D Systems (Minneapolis, MN, USA), and FITC- or PE-labeled monoclonal antibodies specific for CD11c, CD80, CD86 and 4-1BB were purchased from BD Pharmingen (San Diego, CA, USA). Rabbit anti-Bcl-2 mAb, rabbit anti-Bcl-xL mAb and hamster IgG isotype control $\mathrm{mAb}$ were purchased from Cell Signaling Technology.

Isolation and maturation of DCs. Mouse DCs were generated from bone marrow suspensions harvested from 6-8-week old C57BL/6 mice according to the literature (12), with slight modifications. Briefly, bone-marrow cells were harvested from femurs and tibias, depleted of red blood cells and washed twice 
in phosphate-buffered saline (PBS). Cells were resuspended in a DC medium consisting of RPMI-1640 supplemented with $10 \%$ heat-inactivated FCS (Gibco, USA), $10 \mathrm{ng} / \mathrm{ml}$ GM-CSF (R\&D Systems), 10 ng/ml IL-4 (R\&D Systems), 50 mM 2-mercaptoethanol, $100 \mathrm{IU} / \mathrm{ml}$ penicillin and $100 \mu \mathrm{g} / \mathrm{ml}$ streptomycin. The cells were then cultured $\left(37^{\circ} \mathrm{C}, 5 \% \mathrm{CO}_{2}\right)$ in 6 -well plates at $1 \times 10^{6}$ cells $/ 3 \mathrm{ml} /$ well. On days 3 and 5 of culture, floating cells were gently removed, and fresh $\mathrm{mGM}-\mathrm{CSF} / \mathrm{mIL}-4$-containing medium was added. On day 6, non-adherent cells and loosely adherent proliferating DC aggregates were collected. Mature DCs were generated by the addition of $10 \mathrm{ng} / \mathrm{ml}$ LPS (Sigma) for a further $24 \mathrm{~h}$ of culture. The mature DCs were then cultured in medium with $100 \mu \mathrm{g}$ anti-4-1BB Ab, hamster IgG isotype control $\mathrm{Ab}$ or with no added antibody for another $48 \mathrm{~h}$ for the subsequent experiments.

Surface marker analysis of DCs. For phenotypic analyses by flow cytometry, 4-1BB Ab-treated DCs $\left(5 \times 10^{5}\right)$ were stained for 30 min on ice with FITC- or PE-labeled monoclonal antibodies specific for CD11c, CD80 and CD86 (BD Pharmingen). After washing three times in PBS, the cells were analyzed by flow cytometry. Isotype-matched monoclonal antibodies were used as controls.

Cytokine production by DCs. For the cytokine assays, culture supernatants were harvested and used for the enzyme-linked immunosorbent assay (ELISA). Mouse IL-6 and IL-12 Quantikine ELISA Kit (R\&D Systems) were used to detect IL-6 and IL-12, respectively, following the manufacturer's instructions.

Apoptosis analysis by flow cytometry. For the apoptosis analysis, 4-1BB Ab-treated DCs $\left(5 \times 10^{5}\right)$ were collected, and staining was performed using FITC-conjugated annexin $\mathrm{V}$ and propidium iodide $(\mathrm{PI})$ according to the manufacturer's instructions. Apoptosis was analyzed by flow cytometry (Apoptosis Kit, BD Pharmingen, Germany).

Western blot analysis. DCs were collected and lysed. The lysates were separated on 10\% SDS-PAGE. Following electrophoresis, the protein blots were transferred onto a nitrocellulose membrane (Amersham, Waukesha, WI, USA). The membrane was blocked with $5 \%$ non-fat milk in TBST for $1 \mathrm{~h}$ and incubated overnight with rabbit anti-Bcl-2 or rabbit anti-Bcl-xL mAb at $4^{\circ} \mathrm{C}$. After three washes with TBST, the membrane was incubated at $37^{\circ} \mathrm{C}$ for $1 \mathrm{~h}$ with horseradish peroxidase-conjugated goat anti-rabbit IgG secondary antibody diluted with TBST. The detected protein signals were visualized using an enhanced chemiluminescence reaction system. Western blotting for $\beta$-actin was used as an internal sample.

Proliferation assay. Mixed leukocyte reaction (MLR) was performed using three types of mature DCs (anti-4-1BB Ab-treated DCs, hamster IgG isotype control Ab-treated DCs and untreated control DCs) as stimulator cells and T lymphocytes as responder cells. Nylon wool-purified naive T cells derived from the spleen of allogeneic BALB/c mice were plated onto a 96-well round-bottomed culture plate (Costar, USA) at $4 \times 10^{5}$ cells per well. Stimulators were then added and co-cultured with responders at ratios of $1: 10,1: 10^{2}, 1: 10^{3}$ and $1: 10^{4}$ in complete RPMI-1640 medium. DCs and T cells incubated in medium alone served as the stimulator and responder controls, respectively. Following incubation for 4 days, $10 \mu \mathrm{l}$ Cell Counting Kit-8 (Dojindo, Japan) solution was added to each well containing $100 \mu \mathrm{l}$ medium for $4 \mathrm{~h}$. Absorbance was measured at $450 \mathrm{~nm}$ on an automatic ELISA reader (Triturus). All determinations were carried out in triplicate and repeated three times.

Statistical analysis. SPSS 13.0 was used for data variation analysis. Data were presented as the means \pm SD and were analyzed by ANOVA or the Student's t-test. $\mathrm{P}<0.05$ was considered to indicate statistical significance.

\section{Results}

Phenotype analysis of DCs. To characterize the expression of 4-1BB on DCs and the effect of 4-1BB-mediated signaling on co-stimulatory molecules on DCs, bone marrow-derived DCs were analyzed after co-staining for CD11c, CD80, CD86 and 4-1BB. The results showed that mature DCs expressed high levels of $4-1 \mathrm{BB}$, and that the agonistic anti-4-1BB mAb triggered a high expression of CD80 and CD86 (Fig. 1).

Cytokine production by DCs. To determine the mechanism of affection of DCs induced by 4-1BB-mediated signaling, we analyzed the cytokine production of the DCs. Mature DCs were cultured in medium with $100 \mu \mathrm{g}$ anti-4-1BB Ab, hamster IgG isotype control $\mathrm{Ab}$ or with no added antibody for $48 \mathrm{~h}$. The culture supernatants were collected and analyzed for the production of IL- 6 and IL-12 by ELISA. The levels of IL-6 and IL-12 in 4-1BB Ab-treated supernatant were greater than those in the remaining two groups (Fig. 2).

Analysis of DC apoptosis. To detect whether 4-1BB signaling was involved in the survival of DCs, anti-4-1BB Ab-treated, hamster IgG isotype control Ab-treated and untreated mature DCs were collected for the detection of apoptosis by staining with FITC-conjugated annexin V and PI. The rate of apoptosis of anti-4-1BB Ab-treated DCs was lower than that of IgG isotype control Ab-treated DCs and untreated DCs (Fig. 3).

Western blot analysis. To determine the effect of 4-1BB signaling involved in the anti-apoptotic molecules of mature DCs, the DCs were collected and equal amounts of cell lysates were applied to the Western blot analysis of Bcl-2 and Bcl-xL. The two anti-apoptotic proteins were detected in 4-1BB-mediated DCs, and their levels were slightly increased following treatment with anti-4-1BB mAb (Fig. 5).

Proliferation. DCs are potent stimulators of primary MLRs and are capable of inducing the proliferation of allogeneic $\mathrm{T}$ lymphocytes in vitro. We compared the abilities of our DC populations to stimulate primary MLRs among allogeneic T lymphocytes. The data showed that DCs treated with anti4-1BB mAb induced stronger allogeneic $\mathrm{T}$ cell proliferative responses in vitro than untreated DCs and DCs treated with IgG isotype control Ab (Fig. 5). 
A

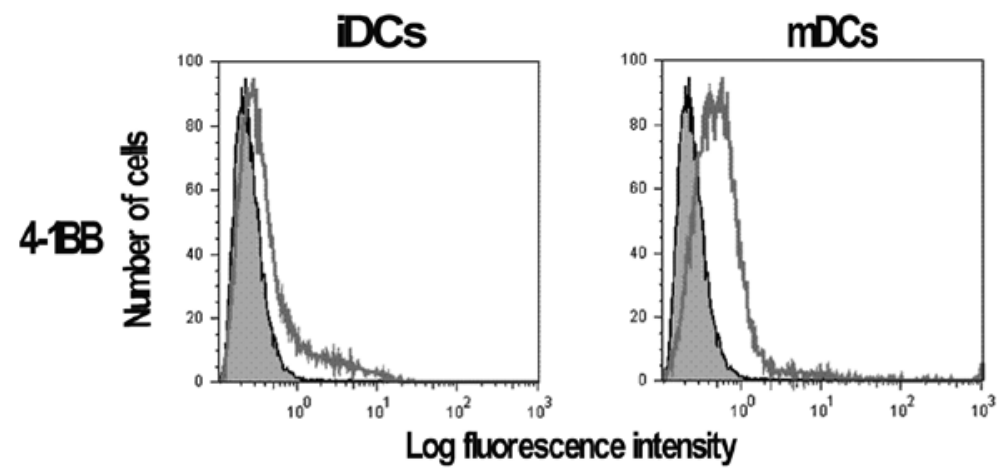

B

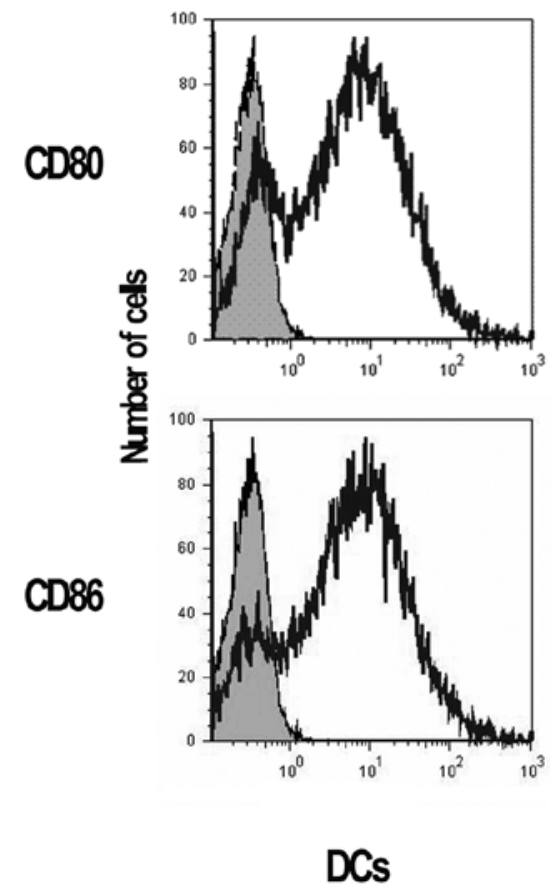

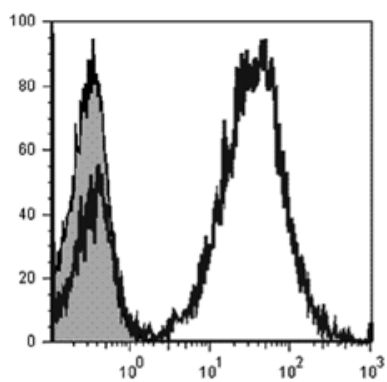
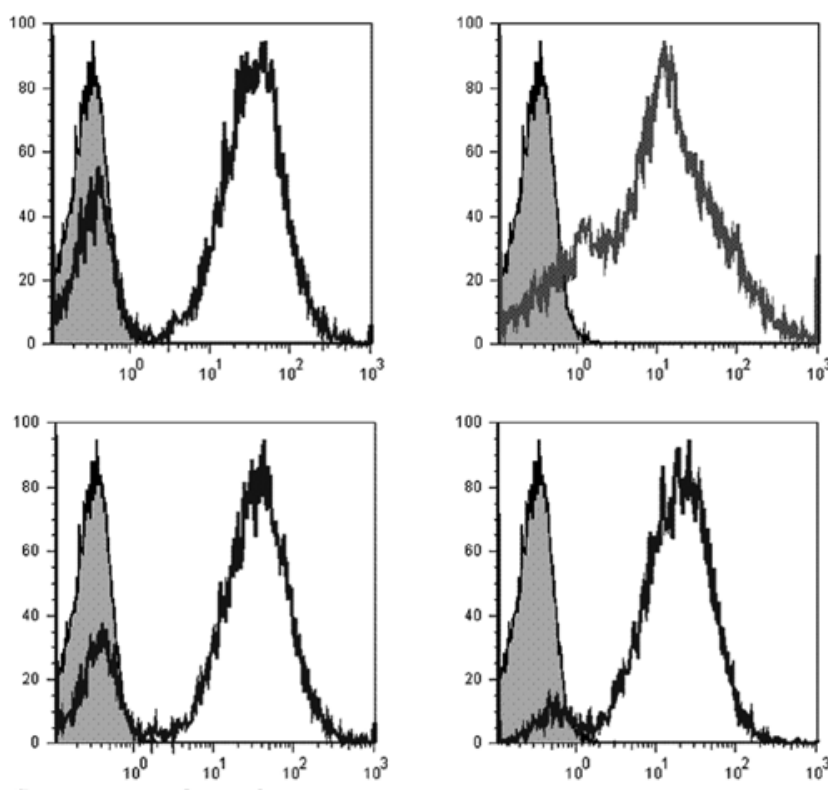

Log fluorescence intensity

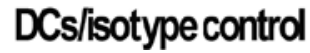

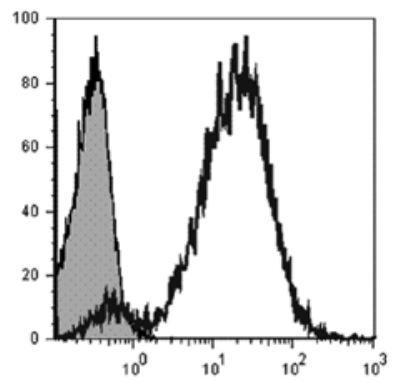

DCs/4-BB Ab

Figure 1. Phenotype analysis of DCs. (A) Immature and LPS (10 ng/ml)-matured DCs were stained with anti-4-1BB (bold histograms) or isotype control mAb (gray histograms). (B) Anti-4-1BB Ab-treated, hamster IgG isotype control Ab-treated and untreated mature DCs were stained with anti-CD80 and CD86 (bold histograms) or isotype control mAb (gray histograms).
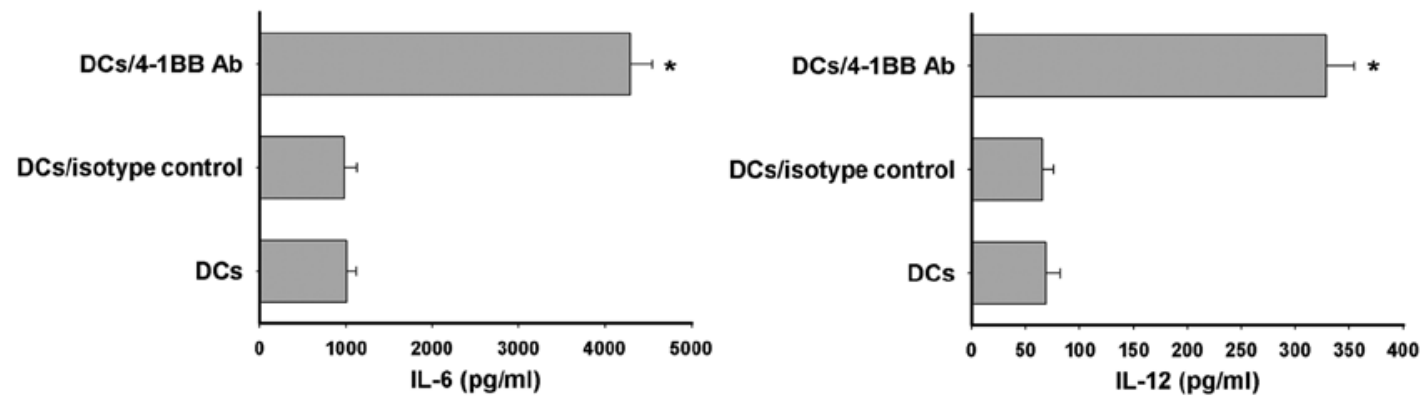

Figure 2. Cytokine (IL-6/IL-12) production by DCs. Mature DCs were cultured in medium with anti-4-1BB Ab, hamster IgG isotype control Ab or with no added antibody for $48 \mathrm{~h}$. The culture supernatants were then collected to analyze the production of IL-6 and IL-12 by ELISA. Data were presented as the means \pm SD. Similar results were obtained from three independent experiments $\left({ }^{*} \mathrm{P}<0.05\right)$.

\section{Discussion}

DCs are one of the most potent APCs for the induction of antitumor immune responses currently known and have been recognized as potentially significant tools for $\mathrm{T}$ cell-mediated anti-cancer immunotherapy (13). 4-1BB is a TNFR superfamily member that has been investigated for its role as a co-stimulatory molecule for $\mathrm{T}$ cells and has been applied in the form of agonistic anti-4-1BB mAb or recombinant 4-1BB ligand protein to strengthen immune responses against viruses 
DCs

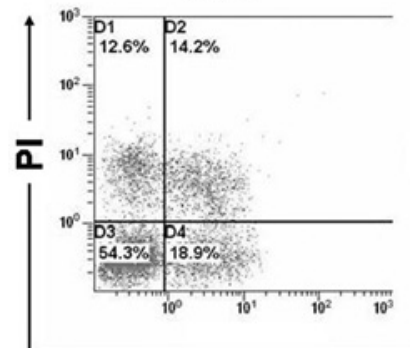

DCs/isotype control

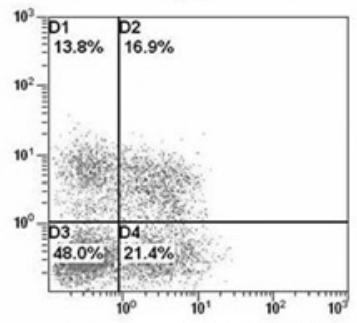

Annexin V-FITC
DCs/4-1BB Ab

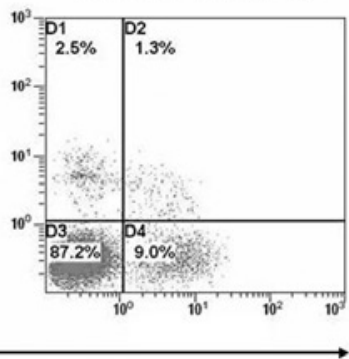

Figure 3. Apoptosis analysis of DCs. Anti-4-1BB Ab-treated, hamster IgG isotype control Ab-treated and untreated mature DCs were collected for the detection of apoptosis by staining with FITC-conjugated annexin V and PI. The results are representative of three independent experiments.

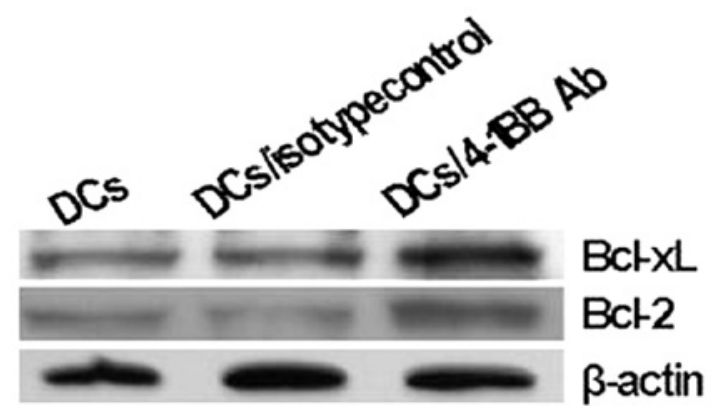

Figure 4. Analysis of the anti-apoptotic molecules of DCs. Mature DCs were cultured in medium with anti-4-1BB Ab, hamster IgG isotype control Ab or with no added antibody for $72 \mathrm{~h}$. DCs were collected, and equal amounts of cell lysates were applied to the western blot analysis of Bcl-2 and Bcl-xL.

and tumors, which eventually increases the activity of $\mathrm{T}$ cells $(11,14)$. However, the function of $4-1 B B$ on DCs remains insufficiently characterized. In the present study, we used an agonistic $\mathrm{mAb}$ against $4-1 \mathrm{BB}$ to investigate the function of 4-1BB on murine DCs.

The results of this study showed that both the immature and mature bone marrow-derived DCs that had been cultured in the presence of LPS expressed 4-1BB, and that the expression level of 4-1BB on mature DCs was higher than that on immature DCs, as shown in Fig. 1A. Moreover, the 4-1BB molecules expressed on DCs were capable of activating DCs, resulting in higher levels of IL- 6 and IL-12 production and the upregulation of CD80 and CD86 (Figs. 1 and 2). It was reported that the linkage of 4-1BB on T cells with its ligand recruited TNFRassociated factor- 2 and resulted in the activation of p38 MAPK, apoptosis signal-regulating kinase-1, and c-Jun N-terminal/ stress-activated protein kinases $(15,16)$, which presumably increase the production of cytokines and the expression of cell surface molecules. Our results suggest that cytokines such as IL-6 and IL-12 play a critical role in this process.

The results presented in this study demonstrate that 4-1BB signaling also functioned as the DC survival signal, for the rate of apoptosis of anti-4-1BB Ab-treated DCs was lower than that of IgG isotype control Ab-treated DCs and untreated DCs (Fig. 3), which might be due to the increased expression of Bcl-2 and Bcl-xL (Fig. 4). Given the significance of DCs in the induction of a $\mathrm{T}$-cell immune response, we aimed to

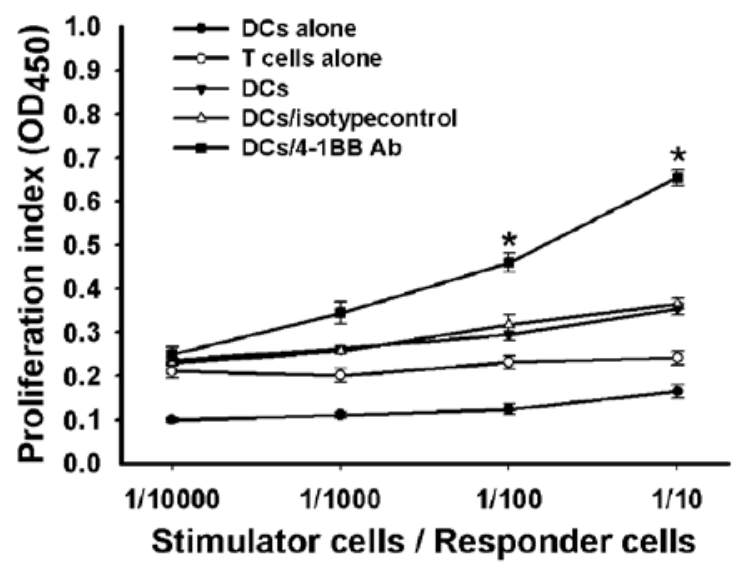

Figure 5. Mixed lymphocyte reaction. T lymphocytes stimulated by anti4-1BB Ab-treated DCs, hamster IgG isotype control Ab-treated DCs, and untreated DCs. The results are presented as the means \pm SD of three replicates. The data show that anti-4-1BB Ab-treated DCs were potent stimulators of lymphocyte compared with hamster IgG isotype control Ab-treated DCs and untreated DCs $\left({ }^{*} \mathrm{P}<0.05\right)$.

determine whether signaling through DC-associated 4-1BB in vitro was able to enhance their T-cell stimulatory function. As shown, DCs treated with anti-4-1BB mAb induced stronger allogeneic T-cell proliferative responses in vitro than untreated DCs and DCs treated with IgG isotype control Ab (Fig. 5). Melero et al (11) reported that the systemic treatment of $\mathrm{mAb}$ against 4-1BB eliminated established tumors in mice by the potent amplification of tumor-specific CD8 ${ }^{+} \mathrm{CTL}$ activity. Our findings suggest that 4-1BB signaling on the DCs may partially explain the potent effect of anti-4-1BB mAb on the activation of tumor-specific CTL. The results of the present study may have profound implications for both our understanding of DC immunobiology and our mechanistic understanding of 4-1BBbased immunotherapy.

\section{Acknowledgements}

This study was supported by the National Natural Science Foundation of China (grant no. 30672107) and the Scholarship Award for Excellent Doctoral Student granted by the Ministry of Education. 


\section{References}

1. Nencioni A, Grünebach F, Schmidt SM, et al: The use of dendritic cells in cancer immunotherapy. Crit Rev Oncol Hematol 65: 191-199, 2008.

2. Palucka K, Ueno H, Fay J and Banchereau J: Dendritic cells and immunity against cancer. J Intern Med 269: 64-73, 2011.

3. Nopora A and Brocker T: Bcl-2 controls dendritic cell longevity in vivo: J Immunol 169: 3006-3014, 2002.

4. Vinay DS and Kwon BS: Differential expression and costimulatory effect of 4-1BB (CD137) and CD28 molecules on cytokine-induced murine CD8(+) Tc1 and Tc2 cells. Cell Immunol 192: 63-71, 1999.

5. Laderach D, Movassagh M, Johnson A, Mittler RS and Galy A: 4-1BB co-stimulation enhances human CD8(+) T cell priming by augmenting the proliferation and survival of effector CD8(+) T cells. Int Immunol 14: 1155-1167, 2002.

6. Lu ZY, Condomines M, Tarte K, et al: B7-1 and 4-1BB ligand expression on a myeloma cell line makes it possible to expand autologous tumor-specific cytotoxic T cells in vitro. Exp Hematol 35: 443-453, 2007.

7. Kudo-Saito C, Hodge JW, Kwak H, Kim-Schulze S, Schlom J and Kaufman HL: 4-1BB ligand enhances tumor-specific immunity of poxvirus vaccines. Vaccine 24: 4975-4986, 2006.

8. Xiao H, Huang B, Yuan Y, et al: Soluble PD-1 facilitates 4-1BBL-triggered antitumor immunity against murine $\mathrm{H} 22$ hepatocarcinoma in vivo. Clin Cancer Res 13: 1823-1830, 2007.
9. Lin GH, Liu Y, Ambagala T, Kwon BS, Ohashi PS and Watts TH Evaluating the cellular targets of anti-4-1BB agonist antibody during immunotherapy of a pre-established tumor in mice. PLoS One 5: e11003, 2010

10. Gullo C, Koh LK, Pang WL, Ho KT, Tan SH and Schwarz H Inhibition of proliferation and induction of apoptosis in multiple myeloma cell lines by CD137 ligand signaling. PLoS One 5: e10845, 2010

11. Melero I, Shuford WW, Newby SA, et al: Monoclonal antibodies against the 4-1BB T-cell activation molecule eradicate established tumors. Nat Med 3: 682-685, 1997.

12. Nagaraj S, Pisarev V, Kinarsky L, et al: Dendritic cell-based full-length survivin vaccine in treatment of experimental tumors. J Immunother 30: 169-179, 2007.

13. O'Neill DW: Dendritic cells and T cells in immunotherapy. J Drugs Dermatol 9: 1383-1892, 2010.

14. Bertram EM, Dawicki W and Watts TH: Role of T cell costimulation in anti-viral immunity. Semin Immunol 16: 185-196, 2004.

15. Cannons JL, Choi Y and Watts TH: Role of TNF receptorassociated factor 2 and p38 mitogen-activated protein kinase activation during 4-1BB-dependent immune response. J Immunol 165: 6193-6204, 2000

16. Cannons JL, Hoeflich KP, Woodgett JR and Watts TH: Role of the stress kinase pathway in signaling via the T cell costimulatory receptor 4-1BB. J Immunol 163: 2990-2998, 1999. 